\title{
First- and second-order phase transitions in a driven lattice gas with nearest-neighbor exclusion
}

\author{
Ronald Dickman* \\ Departamento de Física, ICEx, Universidade Federal de Minas Gerais, \\ 30123-970 Belo Horizonte - MG, Brasil
}

(November 1, 2018)

\begin{abstract}
A lattice gas with infinite repulsion between particles separated by $\leq 1$ lattice spacing, and nearest-neighbor hopping dynamics, is subject to a drive favoring movement along one axis of the square lattice. The equilibrium (zero drive) transition to a phase with sublattice ordering, known to be continuous, shifts to lower density, and becomes discontinuous for large bias. In the ordered nonequilibrium steady state, both the particle and order-parameter densities are nonuniform, with a large fraction of the particles occupying a jammed strip oriented along the drive. The relaxation exhibits features reminiscent of models of granular and glassy materials.
\end{abstract}

PACS numbers: 0.50.Ln, 64.60.Ht, 05.40.-a, 05.10.Ln 
The study of simple nonequilibrium lattice hopping models, such as the asymmetric exclusion process (ASEP), has blossomed over the past decade, motivated by studies of traffic and of granular matter [1] [7. In parallel there is continuing interest in driven diffusive systems (DDS): lattice-gases with biased hopping [8 11], originally proposed as models of fast ionic conductors (FIC) [12]. In the most widely studied case of DDS with attractive interactions, application of a driving field E (favoring hopping along one of the principal lattice directions) causes the interface between phases to orient along the field. Since the critical temperature increases with $\mathrm{E}$, the drive may be said to favor ordering.

In DDS with repulsive interactions, which would appear to be more pertinent to ionic conduction [13], one expects the driving field to suppress ordering (preferential occupation of one sublattice). Indeed, the original field theoretic, simulation, meanfield analyses [14, 15], showed that antiferromagnetic order is destroyed generically for a drive $E>2 J, J$ being the magnitude of the nearest-neighbor interaction. More recent simulations [16,[17, using larger lattices, strongly suggest that global antiferromagnetic order is destroyed by any drive, however small. The observation that FIC materials exhibit phase transitions, even in the presence of repulsive shortrange interactions, and growing interest in transport in particle systems, motivate investigation of a driven system with hard-core repulsion.

In this Letter I describe the case $J \rightarrow \infty$, i.e., a lattice gas with occupancy of nearest-neighbor sites excluded (NNE). (The distance between any pair of particles must be $>1$ lattice spacing; there are no other interactions.) Equilibrium properties of this lattice analog of the hard-sphere fluid were studied via series expansion methods in the 1960's, leading to the conclusion that (on bipartite lattices) the system undergoes a continuous phase transition at a critical density $\rho_{c}$, to a state with preferential occupancy of one sublattice; $\rho_{c} \simeq 0.37$ on the square lattice [18 20]. (The chemical potential is the temperaturelike parameter for this entropy-driven phase transition, which appears to fall in the Ising universality class [19].) To study the driven system, I adopt nearest-neighbor hopping dynamics. Each particle has an intrinsic rate of $1 / 4$ for hopping in the $\pm y$ directions, and of $p / 2$ and $(1-p) / 2$ in the $+x$ and $-x$ directions, respectively. Thus $p-1 / 2$ represents the deviation from equilibrium, with $p=1$ corresponding to $E \rightarrow \infty$ in standard DDS. (Note however that in DDS the driving field dominates the interparticle interaction when $E \gg J$, whereas in the present case hard-core repulsion takes precedence over the drive.)

I study the driven NNE lattice gas on a square lattice of $L^{2}$ sites, using periodic boundary conditions in both directions. Initially $N$ particles are thrown at random onto the lattice, respecting the NNE condition; this yields a homogeneous, disordered initial configuration. [The random sequential adsorption (RSA) or "parking" process used to generate the initial state eventually jams, at a mean density of 0.3641 [21]. In practice, one can reach densities up to about 0.38 for $L=100$.] In the dynamics a randomly selected particle is assigned a hopping direction according to the rates given above, and the new position is accepted subject to the NNE condition. $N$ such attempted moves define one time unit. After a transient, whose duration depends on $p, L$, and the density $\rho=N / L^{2}$, the system reaches a steady state in which the current density $j$ (the net flux of particles along the $+x$ direction per site and unit 
time), and other macroscopic properties fluctuate about stationary values. I report results of extensive simulations at $p=1,0.75$, and 0.6 ; some studies at $p=1 / 2$ were also performed to facilitate comparison with equilibrium.

The stationary current density for $p=1$ is shown in Fig. 1. For $\rho \leq 0.25$ there is little dependence on system size; for the lower range of densities $(\rho \leq 0.1)$ the simulation data are in good agreement with a four-site cluster mean-field calculation. But at higher densities $(\rho=0.272,0.265$, and 0.264 for $L=100,150$, and 200, respectively), $j$ suddenly jumps to a lower value, and then continues to decrease smoothly with density. These data suggest a discontinuous phase transition (in the $L \rightarrow \infty$ limit) at a density $\rho_{c} \simeq 0.263$, far below that of the equilibrium critical point. Before analyzing the evidence for this transition in greater detail, some observations are in order.

First, for $p=1$ the system exhibits jammed states: absorbing configurations in which each particle is blocked from moving by its neighbors (thus $j \equiv 0$ ). While such configurations exist even at very low densities (for example, an array of particles occupying second-neighbor sites, forming a rectangle spanning the system), jammed states are not encountered in simulations for densities less than about 0.32, for $L \geq 150$. In particular, jamming is never observed in the vicinity of the transition to the ordered phase. Jamming occurs readily for $\rho \geq 0.34$; in other words, the drive induces jamming at densities well below the RSA limit. The system can jam for $p<1$ as well: while motion against the drive is possible in this case, it appears that particles queue up to form dense configurations whose lifetime, while finite, grows exponentially with system size. For $p=0.75$ and $L=100$, jamming occurs at densities above about 0.37 .

Jammed configurations are familiar from the two species driven lattice gas, with the two kinds of particle driven in opposite directions (and site exclusion the only interaction) [22,9]. In that case jammed states form rather more readily than in the NNE lattice gas (for large driving fields the required density is $\approx 0.25$ ), and are associated with a (possibly discontinuous) phase transition. The existence of a sharp jamming transition in the driven NNE system, of relevance to models of granular flow, will be addressed in future work.

Second, the two- and four-site cluster mean-field theories (MFT) are so highly constrained by the NNE condition that the cluster probabilities are independent of the drive $p$. Pair MFT predicts a continuous transition to a sublattice-ordered state at $\rho_{c}=1 / 4$, while the four-site approximation shown in Fig. 1 gives $\rho_{c}=0.2696$. But since in equilibrium $\rho_{c} \simeq 0.37$, the fact that the kink in the MFT curve falls near the discontinuous transition should be seen as purely fortuitous. Larger clusters, or a spatially extended MFT will be needed to study the driven NNE lattice gas.

The simulations extend to a maximum time of from $\sim 3 \times 10^{6}$ to $\sim 6 \times 10^{7}$ steps, depending on the time required to reach the stationary state. Data for the stationary current and order parameter densities are obtained from histograms for the corresponding quantities, in the final (stationary) stage. Near the transition, at $p=1$ and 0.75, the histograms are bimodal (see Fig. 2).

In equilibrium, the phase transition in the NNE lattice gas is signaled by a nonzero "antiferromagnetic" order parameter $\phi=\left|\rho_{A}-\rho_{B}\right|$, where $\rho_{i}=N_{i} / L^{2}$, with 
$N_{i}$ the number of particles in sublattice $i$. Fig. 2 shows the evolution of $j(t)$ and $\phi(t)$ in a single trial near $\rho_{c}$ : the order parameter increases suddenly, after a long waiting time, and then jumps between high and low values; the current mirrors the changes in the order parameter. (The time to the onset of ordering varies from trial to trial, within the range $10^{5}-3 \times 10^{6}$, near $\rho_{c}$, for $p=1$ and $L=150$.)

Fig. 3 shows the stationary order parameter as a function of density. The phase transition is clearly discontinuous for $p \geq 0.75$, and continuous for $p=0.6$ and 0.5 (equilibrium). In fact, for $p=0.6$ the system exhibits the hallmarks of a critical point: the variation of the order parameter with $\rho$ becomes sharper with increasing system size; the current varies smoothly with density (Fig. 1, inset); the histograms are unimodal, becoming very broad near the transition, as reflected in a diverging variance of the order parameter (see the lower inset of Fig. 3). There is presumably a tricritical point at some drive $p_{t}$ between 0.6 and 0.75 ; determining the precise value will require more extensive studies of larger systems. The transition from the disordered, high-conductivity state to the ordered, low-conductivity state occurs at lower density, the larger is $p$. Thus we may cross the phase boundary at fixed density by augmenting $p$ (Fig. 3, upper inset); the current, paradoxically, falls sharply in response to an increased drive!

The mechanism of the phase transition becomes clearer when we examine particle configurations. Fig. 4, a typical stationary ordered configuration for $p=1$, reveals a strongly nonuniform distribution: a high-density strip has formed parallel to the drive. Within the strip, all particles occupy a single sublattice, and their movement is blocked. (Comparison of configurations at different times shows that while there are changes on the fringe, the interior of the strip is frozen on time scales of at least $10^{4}$ steps.) The low-density region outside the strip shows no sublattice ordering, and harbors all the mobile particles.

For $p=1$ and 0.75 , the strip often contains a central core of maximum density, while the surrounding particles tend to fall along diagonal "branches" terminating on the core, forming a herringbone pattern, or, as it were, an arrow pointing along the drive. This suggests that the core forms first, and that particles are subsequently trapped along the branches. The growth of the latter is limited by reduction of the density outside the strip. For $p=0.6$ diagonal branches are again visible, but they are broader (5-10 lattice spacings). A very simple model of branch dynamics (via addition or loss of particles at the tip outside the core, using a two-site MFT) yields growth for densities greater than $0.19,0.26$, and 0.29 in the neighborhood of the tip, for $p=1,0.75$, and 0.6 , respectively. (For smaller densities the tip shrinks.)

Fig. 5 shows typical density and order-parameter profiles perpendicular to the drive. The marked variation in the density suggests that a second order parameter (the difference between the maximium and minimum densities, $\Delta \rho=\rho_{\max }-\rho_{\min }$ ), can be associated with the phase transition. This density difference is the order parameter in standard DDS (attractive NN interactions), but in that case the equilibrium system also shows phase separation, whereas the equilibrium NNE lattice gas does not have high- and low-density phases. In the NNE system a nonzero $\Delta \rho$ is a uniquely nonequilibrium effect.

For $p=1$, ordered-state density profiles have $\rho_{\max } \simeq 0.35$, essentially indepen- 
dent of $\rho$, while $\rho_{\min }$ generally falls the range $0.18-0.20$, again without a systematic $\rho$-dependence. For $p>1 / 2$, but less than unity, ordered configurations again show a high-density strip coexisting with a low-density region having a nonzero current and no sublattice ordering. For $p=0.75 \mathrm{I}$ find $\rho_{\max } \simeq 0.383$, while for $p=0.6$, $\rho_{\max } \simeq 0.40 . \rho_{\min }$ also increases with decreasing $p: \rho_{\min } \simeq 0.19,0.253$, and 0.285 for $p=1,0.75$, and 0.6 , resp., in reasonable agreement with the branch-growth model mentioned above. (MFT and numerical details will be reported elsewhere [23].)

Recall that for $p=1$, jamming occurs readily for $\rho \simeq 0.34$, close to the density $\rho_{\max }$ of the jammed strip in the ordered state. Similarly, for $p=0.75$ the density in the strip is about 0.38 , close to the global jamming density of 0.37 or so. (For $p=0.6$ the jamming density, presumably around 0.40 , is beyond the density that can be prepared via RSA.) The strip, then, appears to represent an instability to local jamming in a system whose density lies below that needed for global jamming. It is well known that driven particle systems such as the ASEP can exhibit a shock, i.e., a discontinuous density profile, which back-propagates (in the direction opposite the drive) if the current in the high-density region is smaller than in the low-density region [5]. It is reasonable to suppose that such a shock forms in the present system due to a density fluctuation (perhaps as a result of hopping perpendicular to the drive), and that its 'tail' may then grow until it reaches the 'head,' yielding a highdensity ring which becomes the core of the jammed strip.

Once it has formed, additional particles cannot readily enter the jammed region, and so $\rho_{\max }$ does not vary with $\rho$. As the overall density $\rho$ increases, $\phi$ grows (and $j$ decreases) as a result of expansion of the jammed region. Eventually it fills the system; globally jammed configurations have perfect sublattice ordering $(\phi=\rho)$, and a uniform density profile, except for empty triangular or diamond-shaped regions that sometimes appear.

I turn now to some preliminary results on dynamics. The apparent waiting time, $\tau$, to the onset of the stationary state typically falls in the range $10^{4}-10^{5}$ for $\rho<\rho_{c}$. At the transition it shows a sharp maximum, $1-3$ orders of magnitude above the pre-transition value, and then falls off gradually $\left(\tau\left(\rho_{c}\right) \approx 2 \times 10^{6}\right.$ for $p=1$ and $L=150)$. Definitive results on relaxation times will require larger samples than were used in this study. Another interesting observation is the appearance, in some realizations at $p=1$ and $\rho \geq 0.3$, of slow relaxation. The order parameter grows linearly with $\ln t$ over a sizeable interval (e.g., $6 \times 10^{4}-3 \times 10^{6}$ ), before saturating. This is reminiscent of the slow compactification seen in granular materials. A model with purely excluded-volume interactions, but with rather different boundary and driving conditions, has in fact been found to reproduce slow (logarithmic) granular compaction [24]. The present model may also be viewed as a 'scalar' version of the situation envisioned in Ref. [25], in which application of a shear stress provokes jamming (and thus rigidity) in a granular medium.

The mean time $\tau_{J}$ to jamming appears to have an exponential dependence on the density. Studies at $p=1$ and $L=150$ give $\tau_{J} \simeq 10^{4}$ for $\rho \geq 0.34$, while for smaller densities $\tau_{J}$ grows exponentially with $(0.34-\rho)$, increasing by more than two orders of magnitude between $\rho=0.34$ and $0.32 ; \tau_{J}$ exceeds the simulation time for $\rho<0.31$. 
Related to the first-order phase transition is the question of hysteresis, and of the nucleation, growth, and decay of a jammed strip. The nonequilibrium critical behavior observed for a smaller drive (e.g., $p=0.6$ ) is an important subject for detailed study, since the nature of scaling in DDS remains controversial [9, 10, Further issues to be explored in future work are the effects of different initial configurations, of the aspect ratio (in rectangular systems), of boundary conditions (open along the drive, and/or reflecting perpendicular to it), and systematic studies of temporal and spatial correlations, and of finite-size effects. Finally, it would be very useful to develop continuum descriptions of this system, be they stochastic (Langevin-like, starting from a suitable time-dependent Landau-Ginzburg formulation), or deterministic (hydrodynamic, starting perhaps from a kinetic theory of the lattice model).

In summary, I have shown that the phase transition in the lattice gas with nearest-neighbor exclusion persists under a drive, and turns discontinuous for a sufficiently large bias. Ordering is attended by the qualitatively new phenomenon (for a system with repulsive interactions) of phase segregation, which appears to result from an instability to formation of jammed regions. As in the attractive DDS, certain features of the ordered state can be understood on the basis of dynamic stability, as opposed to interactions or free-energy considerations. In the context of ASEP-like models, the results show that a two-dimensional system is capable of exhibiting a bulk phase transition, whereas the corresponding one-dimensional system is expected to show only boundary-induced transitions. The driven NNE lattice gas displays a surprisingly rich variety of behaviors for its simplicity, and may be of relevance to experiments on ionic conductors. Certain aspects of its behavior may yield insights into glassy and granular relaxation.

\section{Acknowledgements}

I thank Robin Stinchcombe, Gyorgy Szabó, and Miguel Angel Muñoz for helpful discussions. This work was supported in part by CNPq and CAPES.

*electronic address: dickman@fisica.ufmg.br 


\section{REFERENCES}

[1] J. Krug, Phys. Rev. Lett. 67, 1882 (1991).

[2] B. Derrida, M. R. Evans, V. Hakim, and V. Pasquier, J. Phys. A 26, 1493 (1993).

[3] R. B. Stinchcombe and G. M. Schütz, Phys. Rev. Lett. 75, 140 (1995); R. B. Stinchcombe, M. D. Grynberg, and M. Barma, Phys. Rev. E 47, 4018 (1993).

[4] D. E. Wolf and M. Schreckenberg (eds.) Traffic and Granular Flow (SpringerVerlag, Berlin, 1998).

[5] G. M. Schütz, in Phase Transitions and Critical Phenomena, C. Domb and J. L. Lebowitz, eds., (Academic Press, London, in press).

[6] H. M. Jaeger, S. R. Nagel, and R. P. Behringer, Rev. Mod. Phys. 68, 1259 (1996).

[7] L. P. Kadanoff, Rev. Mod. Phys. 71, 435 (1999).

[8] S. Katz, J. L. Lebowitz, and H. Spohn, Phys. Rev. B 28, 1655 (1983); J. Stat. Phys. 34, 497 (1984).

[9] B. Schmittmann and R. K. P. Zia, Statistical Mechanics of Driven Diffusive Systems, vol. 17 of Phase Transitions and Critical Phenomena, C. Domb and J. L. Lebowitz, eds., (Academic Press, London, 1995).

[10] J. Marro and R. Dickman, Nonequilibrium Phase Transitions in Lattice Models (Cambridge University Press, Cambridge, 1999).

[11] T. Antal and G. M. Schütz, Phys. Rev. E62, 83 (2000).

[12] J. B. Boyce and B. A. Huberman, Phys. Rep. 51, 189 (1979); W. Dieterich, P. Fulde, and I. Peschel, Adv. Phys. 29, 527 (1980).

[13] While the crystal lattice may mediate an effective attractive contribution to the interaction, excluded-volume and Coulombic forces should in general yield a net repulsion between ions of the same species, at short range.

[14] K.-t. Leung, B. Schmittmann, and R. K. P. Zia, Phys. Rev. Lett. 62, 1772 (1989).

[15] R. Dickman, Phys. Rev. A 41, 2912 (1990).

[16] G. Szabó, A. Szolnoki, and T. Antal, Phys. Rev. E 49, 299 (1994).

[17] G. Szabó and A. Szolnoki, Phys. Rev. E 53, 2196 (1996).

[18] L. K. Runnels and L. L. Combs, J. Chem. Phys. 45, 2482 (1966).

[19] D. S. Gaunt and M. E. Fisher, J. Chem. Phys. 43, 2840 (1965); D. S. Gaunt, ibid. 46, 3237 (1967).

[20] F. H. Ree and D. A. Chesnut, J. Chem. Phys. 45, 3983 (1966).

[21] P. Meakin, J. L. Cardy, E. Loh, and D. J. Scalapino, J. Chem. Phys. 86, 2380 (1987); R. Dickman, J.-S. Wang, and I. Jensen, ibid., 94, 8252 (1991).

[22] B. Schmittmann, K. Hwang, and R. K. P. Zia, Europhys. Lett. 19, 19 (1992).

[23] R. Dickman, to be published.

[24] E. Caglioti, V. Loreto, H. J. Herrmann, and M. Nicodemi, Phys. Rev. Lett. 79, 1575 (1997).

[25] M. E. Cates, J. P. Wittmer, J.-P. Bouchaud, and P. Claudin, Phys. Rev. Lett. 81, 1841 (1998). 


\section{FIGURE CAPTIONS}

FIG. 1. Stationary current density versus particle density for $p=1$ and $L=50$ (+), $L=100$ (o), and $L=150$ (diamonds). Vertical lines indicate the transition at $L=100$ (dotted) and $L=150$ (solid). Solid curve: four-site MFT. Inset: $j$ versus $\rho$ for $p=0.6$ in the region of the transition, $L=100$.

FIG. 2. Order parameter (lower panel) and current density (upper) in a single trial at $p=1, \rho=0.2715$ and $L=100$. Inset: order-parameter histogram for the same run, accumulated for $t>3.3 \times 10^{6}$.

FIG. 3. Stationary order parameter versus density for $p=1$ (squares), $p=0.75$ (circles), and $p=0.6$ (diamonds). Open symbols are for $L=100$, filled, $L=150$. Crosses represent the equilibrium system $(p=1 / 2)$ with $L=100$. Upper inset: estimated phase boundary in the $\rho-p$ plane. Lower inset: $\chi \equiv L^{2} \operatorname{var}(\phi)$ versus density for $p=0.6$ and $L=100$ (open symbols) and 150 (filled symbols).

FIG. 4. Snapshot of particle configuration in stationary state, $p=1, \rho=0.267, L=$ 200. Filled and open symbols represent particles on different sublattices. Driving field toward the right.

FIG. 5. Density (solid line) and order-parameter (broken line) profiles perpendicular to the field for the configuration shown in Fig. 4. 


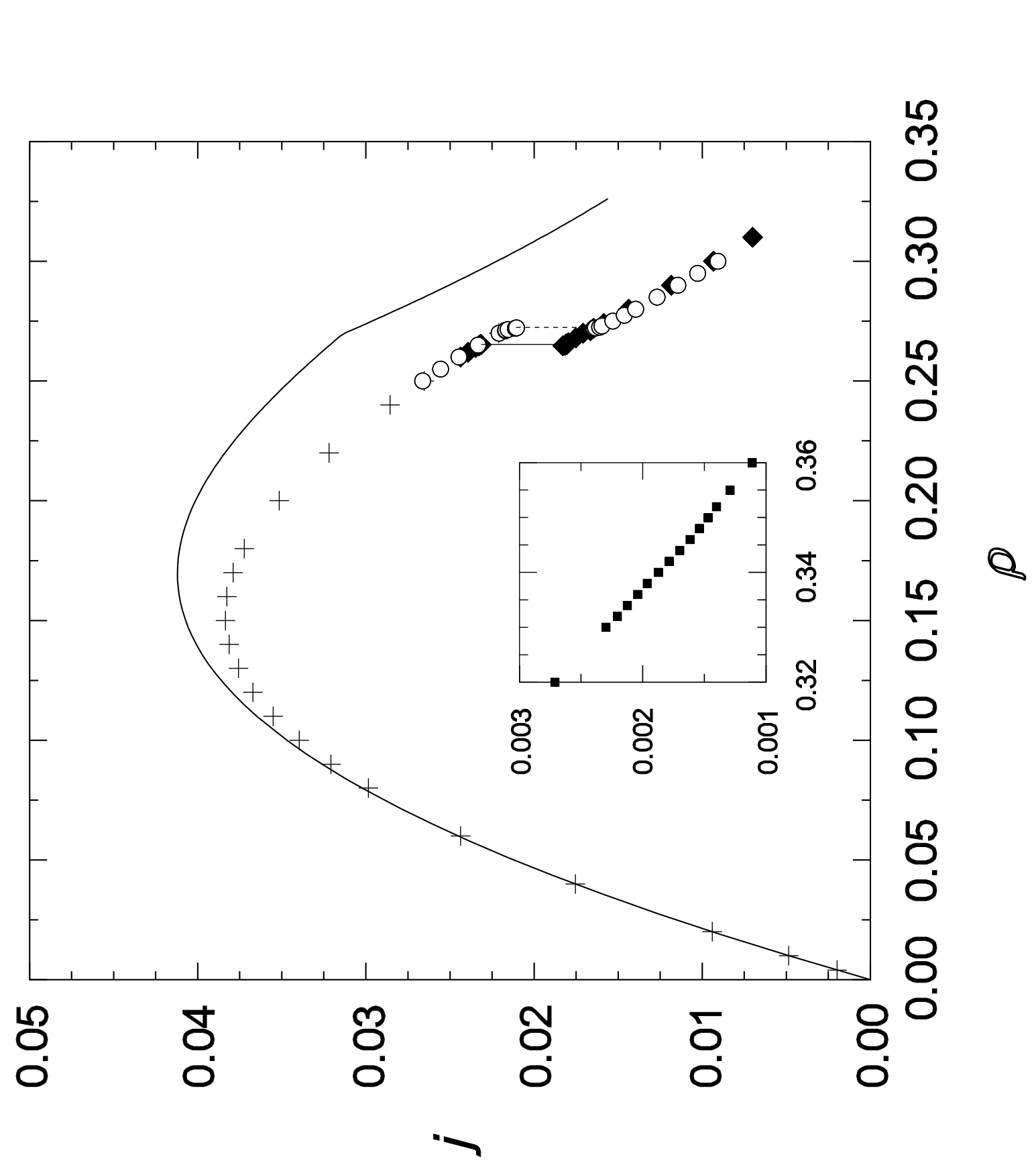

문

$\Omega$ 
N
$\frac{1}{4}$

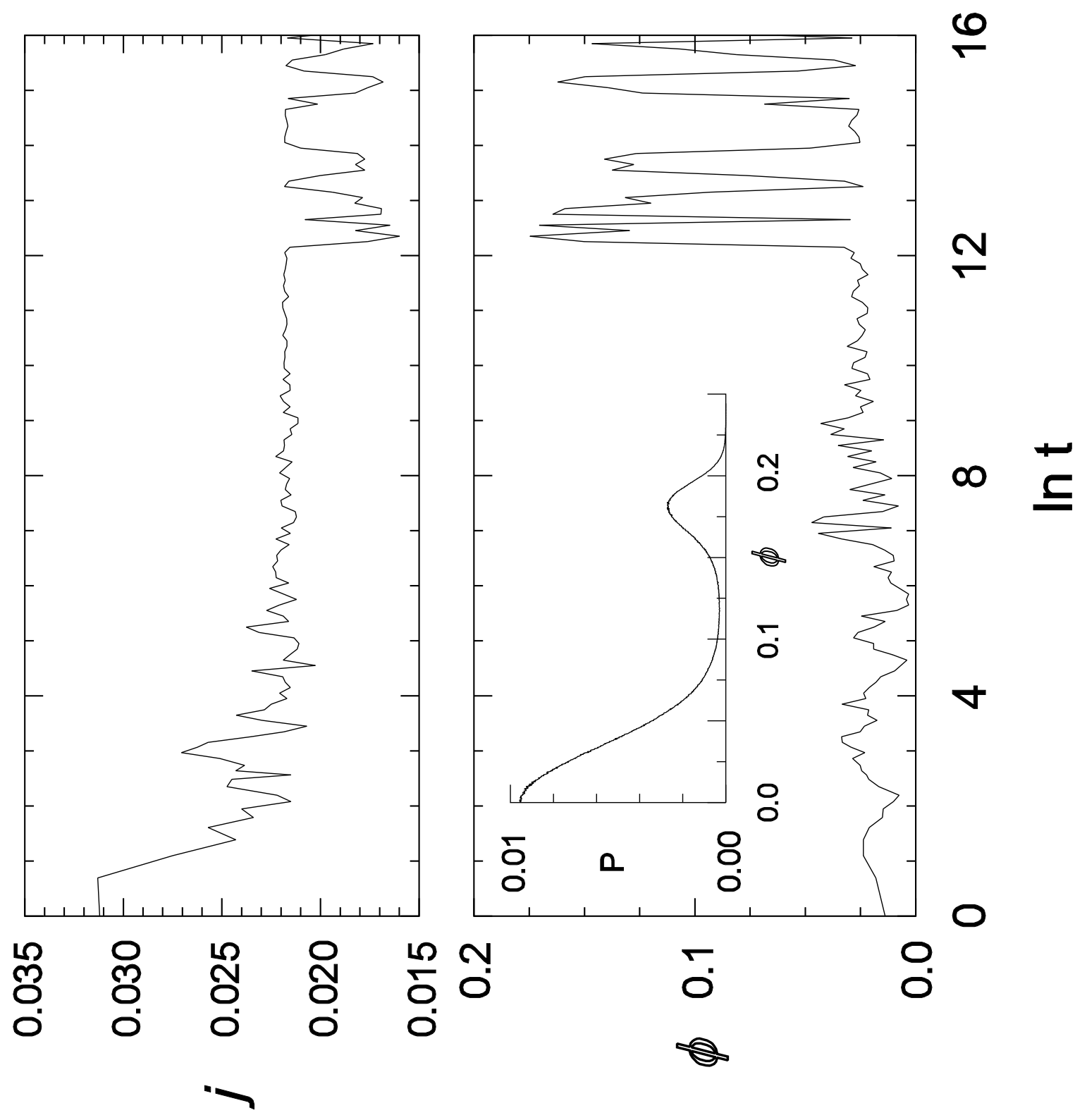


문

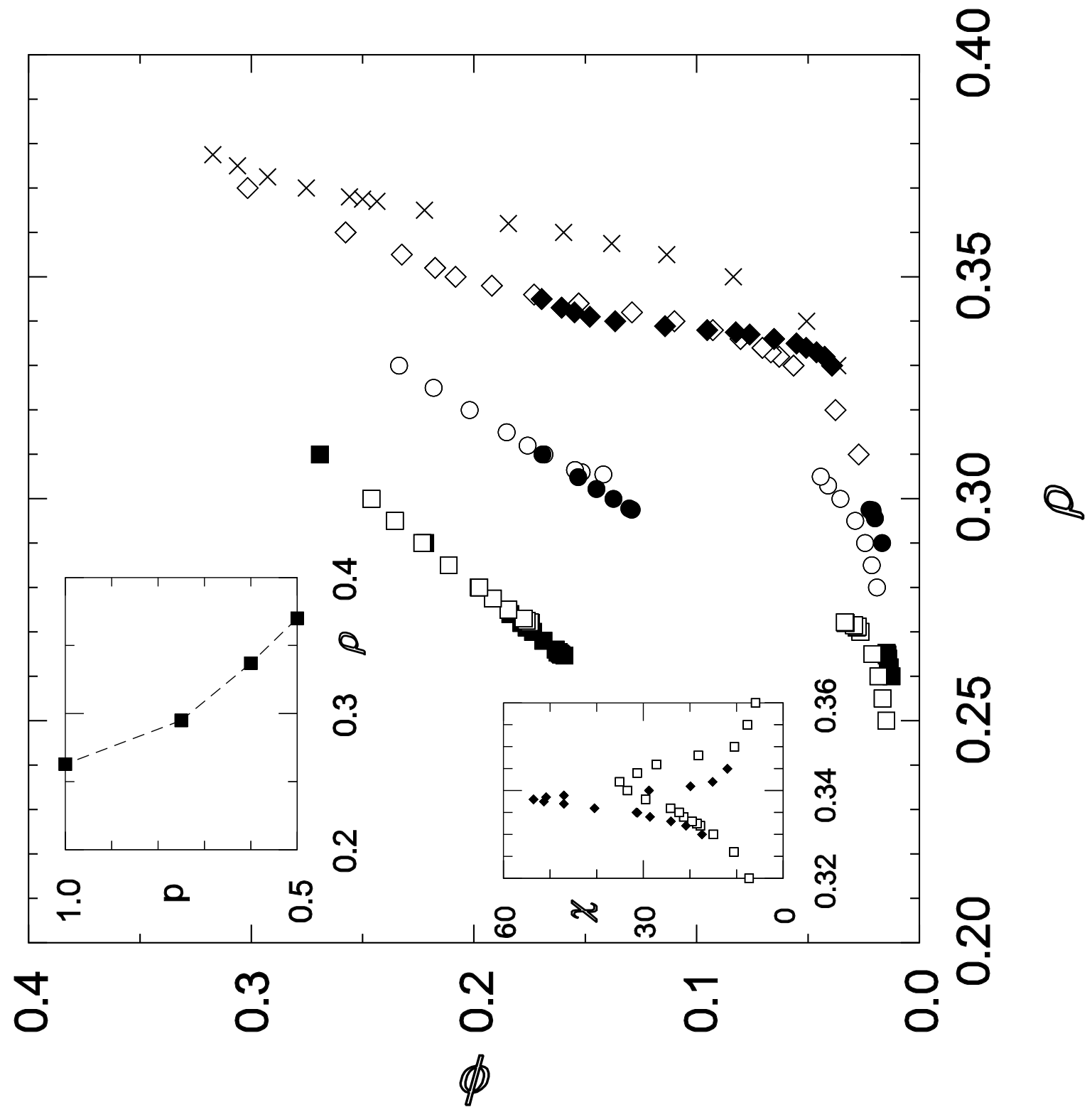




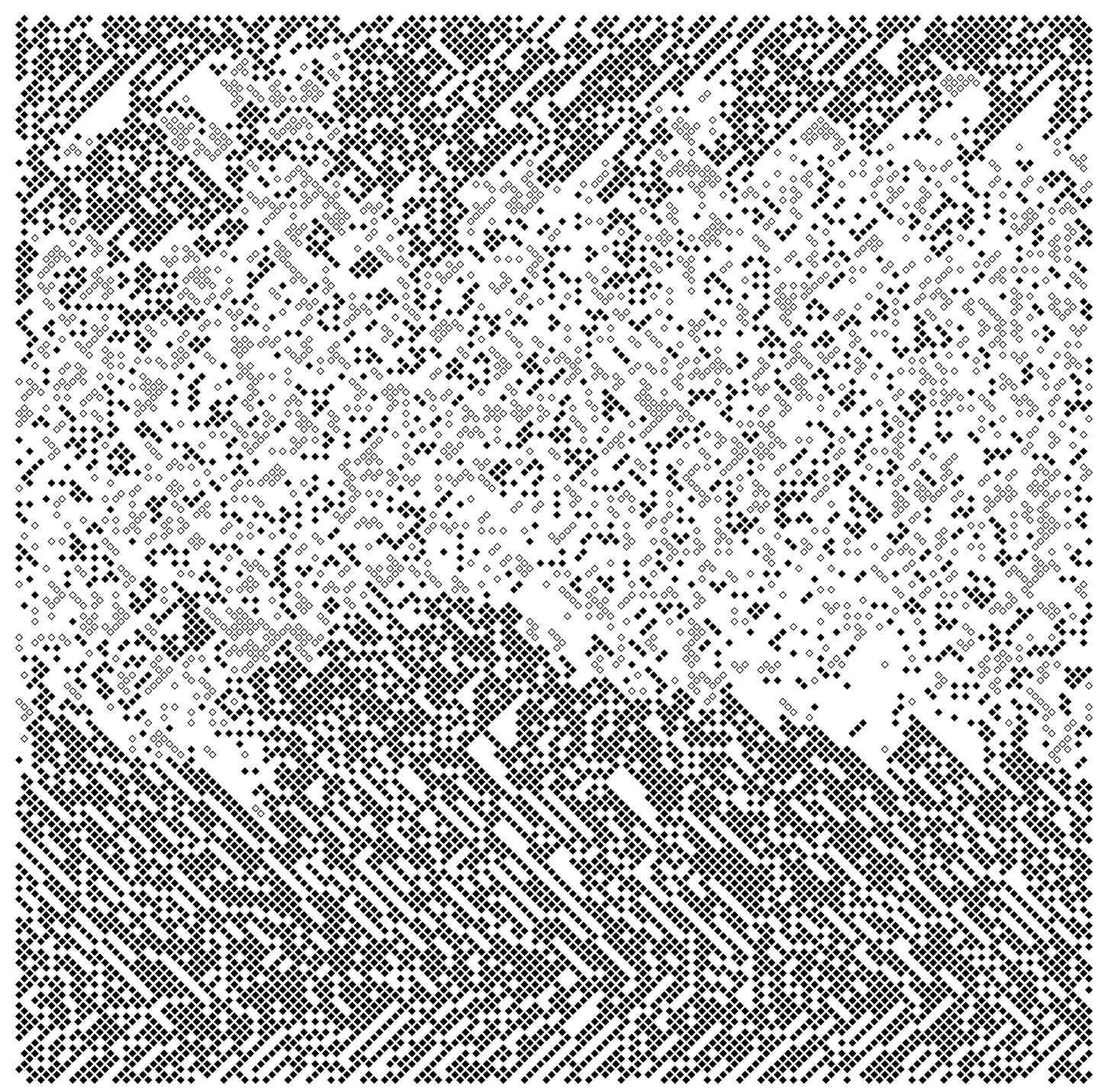


0
$\frac{0}{4}$

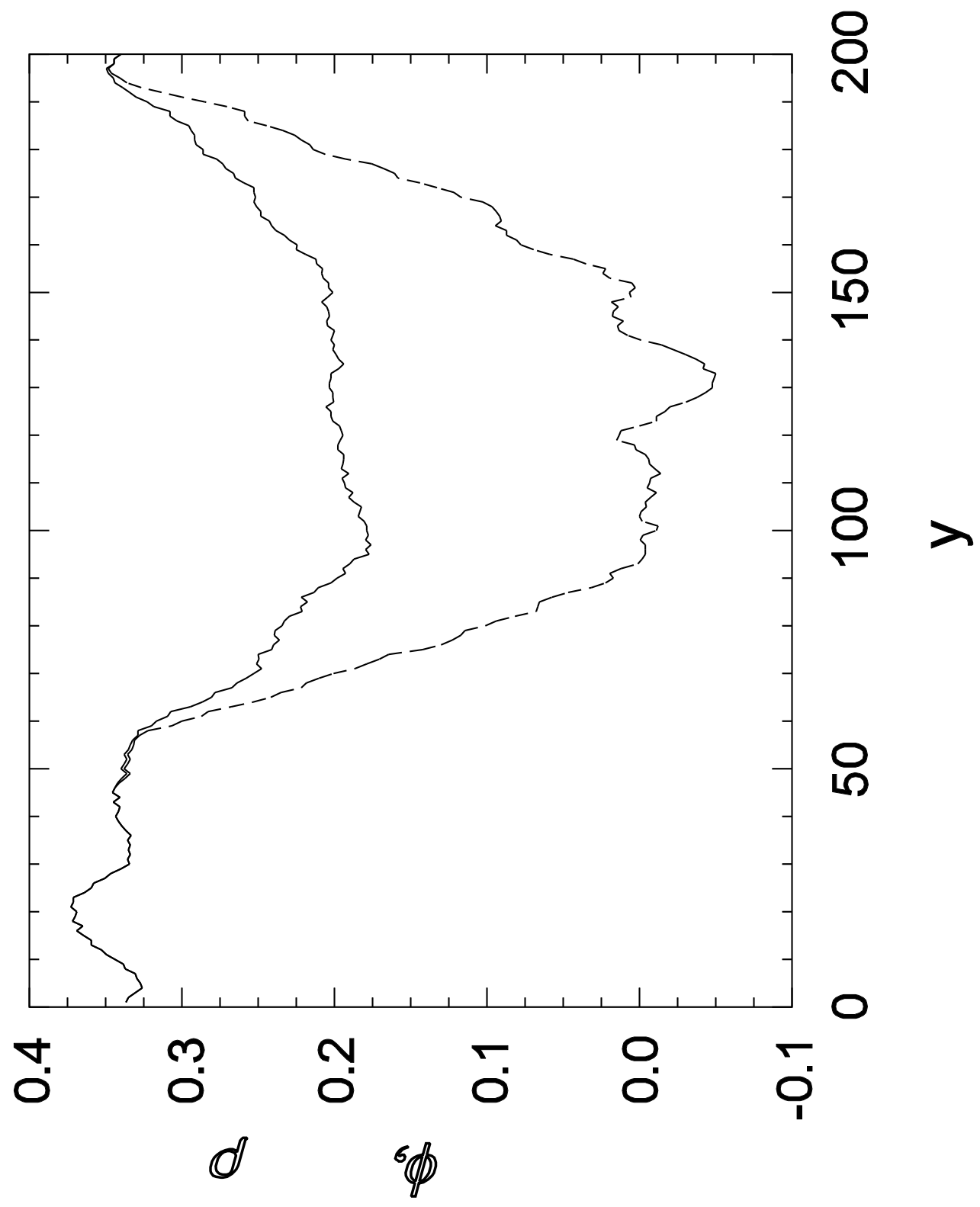

